\title{
Nonoscillation of First-Order Neutral Impulsive Difference Equations
}

\author{
Gokula Nanda Chhatria \\ Sambalpur University \\ Department of Mathematics \\ Sambalpur, 768019, India
}

\begin{abstract}
In this work, we establish sufficient conditions for the existence of nonoscillatory solutions of a class of first-order neutral impulsive difference equations with fixed moments of impulsive effect. The main tools used for the proof of the existence of nonoscillatory solutions are Knaster-Tarski fixed point theorem and Banach's contraction mapping principle.
\end{abstract}

AMS Subject Classifications: 39A10, 39A12.

Keywords: Oscillation, nonoscillation, impulsive difference equation, nonlinear, delay.

\section{Introduction}

Consider the class of first-order impulsive neutral difference equations of the form

$(E)\left\{\begin{array}{l}\Delta[y(n)+p(n) y(n-\tau)]+q(n) G(y(n-\sigma))=0, n \neq m_{j}, j \in \mathbb{N}, \\ \underline{\Delta}\left[y\left(m_{j}-1\right)+p\left(m_{j}-1\right) y\left(m_{j}-\tau-1\right)\right]+r\left(m_{j}-1\right) G\left(y\left(m_{j}-\sigma-1\right)\right)=0,\end{array}\right.$

where $\tau, \sigma>0$ are integers, $p, q, r$ are real valued functions with discrete arguments such that $q(n)>0, r>0,|p(n)|<\infty$ for $n \in \mathbb{N}\left(n_{0}\right)=\left\{n_{0}, n_{0}+1, \cdots\right\}, G \in C(\mathbb{R}, \mathbb{R})$ satisfying the properties $x G(x)>0$ for $x \neq 0$ and $\Delta$ is the forward difference operator defined by $\Delta u(n)=u(n+1)-u(n)$. Let $m_{1}, m_{2}, m_{3}, \cdots$ be the discrete moments of impulsive effect satisfying the property $0<m_{1}<m_{2}<\cdots, \lim _{j \rightarrow \infty} m_{j}=+\infty$. Here, $\underline{\Delta}$ is the difference operator defined by $\Delta y\left(m_{j}-1\right)=y\left(m_{j}\right)-y\left(m_{j}-1\right)$.

In recent years, much effort has been given to study the oscillation and nonoscillation of impulsive differential/difference equations, since it much more richer that the

Received April 18, 2019; Accepted September 5, 2019

Communicated by Tongxing Li 
corresponding theory of differential/difference equations and such equation adequate mathematical modelling of real world phenomena observed in population dynamics, industrial robotics, rhythmical beating, discontinuity of solutions, etc.

In this work, our objective is to study the existence of nonoscillatory solutions of the system $(E)$ when $|p(n)|<\infty$. About the impulsive differential/difference equations we refer the monograph [5] and some of the works [6]- [16] to the reader and the references cited there in.

In [11], Peng has established the oscillation criteria for second order impulsive delay difference equations of the form

$$
\left(E_{1}\right)\left\{\begin{array}{l}
\Delta\left(a_{n-1}|\Delta x(n-1)|^{\alpha-1} \Delta x(n-1)\right)+f(n, x(n), x(n-l))=0, n \neq n_{k} \\
a_{n_{k}}\left|\Delta x\left(n_{k}\right)\right|^{\alpha-1} \Delta x\left(n_{k}\right)=N_{k}\left(a_{n_{k}-1}\left|\Delta x\left(n_{k}-1\right)\right|^{\alpha-1} \Delta x\left(n_{k}-1\right)\right), k \in \mathbb{N}
\end{array}\right.
$$

In [9], Lu et al. have established the oscillation criteria for nonlinear third order difference equations with impulse of the form

$$
\left(E_{2}\right)\left\{\begin{array}{l}
\Delta^{3} y(n)+p(n) f(y(n-\tau))=0, n \neq n_{k} \\
\Delta^{i} y\left(n_{k}\right)=g_{i, k} \Delta^{i} y\left(n_{k}-1\right), i=0,1,2, k \in \mathbb{N}
\end{array}\right.
$$

Unlike the above method, our impulsive effect satisfies another neutral equation corresponding to its difference equation. Inspired and motivated by the above mention works, an attempt is made to find some sufficient condition for nonoscillation of $(E)$ using Knaster-Tarski fixed point theorem and Banach's contraction mapping principle.

Definition 1.1. By a solution of $(E)$ we mean a real valued function $y(n)$ defined on $\mathbb{N}\left(n_{0}-\rho\right)$ which satisfy $(E)$ for $n \geq n_{0}$ with the initial conditions $y(i)=\phi(i), i=$ $n_{0}-\rho, \cdots, n_{0}$, where $\phi(i), i=n_{0}-\rho, \cdots, n_{0}$ are given and $\rho=\max \{\tau, \sigma\}$.

Definition 1.2. A nontrivial solution $y(n)$ of $(E)$ is said to be nonoscillatory, if it is either eventually positive or eventually negative. Otherwise, the solution is said to be oscillatory.

The following fixed point theorem will be used in the proof of main results.

Theorem 1.3 (Knaster-Tarski Fixed Point Theorem, see [5]). Let $X$ be a partially ordered Banach space with ordering $\leq$. Let $S$ be a subset of $X$ with the following properties: the infimum of $S$ belongs to $S$ and every nonempty subset of $S$ has a supremum which belongs to $S$. Let $T: S \rightarrow S$ be a increasing mapping, i.e., $x \leq y$ implies that $T x \leq T y$. Then $T$ has a fixed point in $S$.

Theorem 1.4 (Banach's Contraction Mapping Principle, see [5]). A contraction mapping on a complete metric space has exactly one fixed point. 


\section{Main Results}

\section{Theorem 2.1. Assume that}

$\left(H_{1}\right) \sum_{n=N}^{\infty} q(n)+\sum_{j=1}^{\infty} r\left(m_{j}-1\right)<\infty, N>0$

holds. If one of the following conditions holds:
(A) $0 \leq p_{1} \leq p(n) \leq p_{2}<1$,
(B) $1<p_{3} \leq p(n) \leq p_{4}<\infty$,
$(C)-1<p_{5} \leq p(n) \leq p_{6} \leq 0$,
(D) $-\infty<p_{7} \leq p(n) \leq p_{8}<-1$,

then (E) has a bounded nonoscillatory solution.

Proof. Case-A. $0 \leq p_{1} \leq p(n) \leq p_{2}<1$

Let $X=l_{\infty}^{n_{0}}$ be the Banach space of real valued bounded functions $y(n)$ for $n \geq n_{0}$ with sup norm defined by $\|y\|=\sup \left\{|y(n)|: n \geq n_{0}\right\}$. Set

$$
\mu=\left\{y \in X: \alpha_{1} \leq y(n) \leq \alpha_{2}, n \geq n_{0}\right\}
$$

where $\alpha_{1}$ and $\alpha_{2}$ are two positive constants such that

$$
\alpha_{1}<\left(1-p_{2}\right) \alpha_{2} \text { and } \alpha_{1}+p_{2} \alpha_{2}<\beta<\alpha_{2} \text {. }
$$

Clearly, $\mu$ is a closed subset of $X$ implies that $\mu$ is a complete metric space. From $\left(H_{1}\right)$, it is possible to choose $n_{1}>n_{0}$ such that

$$
\sum_{s=n}^{\infty} q(s)+\sum_{n_{1} \leq m_{j}-1 \leq n} r\left(m_{j}-1\right)<M, n \geq n_{1},
$$

where

$$
M=\min \left\{\frac{\alpha_{2}-\beta}{L}, \frac{\beta-\left(\alpha_{1}+p_{2} \alpha_{2}\right)}{L}, \frac{1-\left(p_{1}+p_{2}\right)}{L}\right\},
$$

and $L=\max \left\{L_{1}, G\left(\alpha_{2}\right)\right\}, L_{1}$ is the Lipschitz constant of $G$ on $\left[\alpha_{1}, \alpha_{2}\right]$. Define a map $\mathcal{T}: \mu \rightarrow \mu$ by

$$
\mathcal{T} y(n)=\left\{\begin{array}{l}
\mathcal{T} y\left(n_{1}+\rho\right), n_{1} \leq n \leq n_{1}+\rho, \\
\beta-p(n) y(n-\tau)+\sum_{s=n}^{\infty} q(s) G(y(s-\sigma)) \\
+\sum_{n_{1} \leq m_{j}-1 \leq n} r\left(m_{j}-1\right) G\left(y\left(m_{j}-\sigma-1\right)\right), n \geq n_{1}+\rho,
\end{array}\right.
$$

where $\rho=\max \{\tau, \sigma\}$. For $y \in \mu$ and using (2.1), we have

$$
\mathcal{T} y(n) \leq \beta+\sum_{s=n}^{\infty} q(s) G(y(s-\sigma))+\sum_{n_{1} \leq m_{j}-1 \leq n} r\left(m_{j}-1\right) G\left(y\left(m_{-} \sigma-1\right)\right)
$$




$$
\begin{aligned}
& \leq \beta+G\left(\alpha_{2}\right)\left[\sum_{s=n}^{\infty} q(s)+\sum_{n_{1} \leq m_{j}-1 \leq n} r\left(m_{j}-1\right)\right] \\
& \leq \beta+G\left(\alpha_{2}\right) M \\
& =\alpha_{2}
\end{aligned}
$$

and

$$
\begin{aligned}
\mathcal{T} y(n) & \geq \beta-p(n) y(n-\tau) \\
& \geq \alpha_{1}+p_{2} \alpha_{2}-p_{2} \alpha_{2} \\
& =\alpha_{1}
\end{aligned}
$$

implies that $\mathcal{T} y \in \mu$ for every $n \geq n_{1}$. In order to apply the Banach's contraction principle we have to show that $\mathcal{T}$ is a contraction mapping on $\mu$. For $y_{1}, y_{2} \in \mu$, we have

$$
\begin{aligned}
\left|\mathcal{T} y_{1}(n)-\mathcal{T} y_{2}(n)\right| & \leq|P(n)|\left|y_{1}(n-\tau)-y_{2}(n-\tau)\right| \\
& +L_{1} \sum_{s=n}^{\infty} q(s)\left|y_{1}(s-\sigma)-y_{2}(s-\sigma)\right| \\
& +L_{1} \sum_{n_{1} \leq m_{j}-1 \leq n} r\left(m_{j}-1\right)\left|y_{1}\left(m_{j}-\sigma-1\right)-y_{2}\left(m_{j}-\sigma-1\right)\right|,
\end{aligned}
$$

that is,

$$
\begin{aligned}
\left|\mathcal{T} y_{1}(n)-\mathcal{T} y_{2}(n)\right| & \leq p_{2}\left\|y_{1}-y_{2}\right\|+L_{1}\left\|y_{1}-y_{2}\right\|\left[\sum_{s=n}^{\infty} q(s)+\sum_{n_{1} \leq m_{j}-1 \leq n} r\left(m_{j}-1\right)\right] \\
& \leq\left[p_{2}+1-\left(p_{1}+p_{2}\right)\right]\left\|y_{1}-y_{2}\right\|
\end{aligned}
$$

implies that

$$
\left\|\mathcal{T} y_{-} \mathcal{T} y_{2}\right\| \leq \lambda\left\|y_{1}-y_{2}\right\|
$$

Therefore, $\mathcal{T}$ is a contraction with $\lambda=1-p_{1}<1$. Hence, by Banach's fixed point theorem, $\mathcal{T}$ has a unique point $y \in \mu$ such that $\mathcal{T} y=y$. Therefore,

$$
y(n)=\left\{\begin{array}{l}
y\left(n_{1}+\rho\right), n_{1} \leq n \leq n_{1}+\rho, \\
\beta-p(n) y(n-\tau)+\sum_{s=n}^{\infty} q(s) G(y(s-\sigma)) \\
+\sum_{j=1}^{\infty} r\left(m_{j}-1\right) G\left(y\left(m_{j}-\sigma-1\right)\right), n \geq n_{1}+\rho
\end{array}\right.
$$

and it is easy to see that $y(n)$ is a nonoscillatory solution of $(E)$.

Case-B. $1<p_{3} \leq p(n) \leq p_{4}<\infty$ 
Let $X=l_{\infty}^{n_{0}}$ be the Banach space of real valued bounded functions $y(n)$ for $n \geq n_{0}$ with sup norm defined by $\|y\|=\sup \left\{|y(n)|: n \geq n_{0}\right\}$. Set

$$
\mu=\left\{y \in X: \alpha_{3} \leq y(n) \leq \alpha_{4}, n \geq n_{0}\right\},
$$

where $\alpha_{3}$ and $\alpha_{4}$ are two positive constants such that

$$
p_{4} \alpha_{3}<\left(p_{3}-1\right) \alpha_{4} \quad \text { and } \quad \alpha_{4}+p_{4} \alpha_{3}<\beta<p_{3} \alpha_{4} .
$$

Since $\mu$ is a closed subset of $X$ then $\mu$ is a complete metric space. From $\left(H_{1}\right)$, it is possible to choose $n_{1}>n_{0}$ such that

$$
\sum_{s=n}^{\infty} q(s)+\sum_{n_{1} \leq m_{j}-1 \leq n} r\left(m_{j}-1\right)<M, n \geq n_{1},
$$

where

$$
M=\min \left\{\frac{p_{3} \alpha_{4}-\beta}{L}, \frac{\beta-\left(\alpha_{4}+p_{4} \alpha_{3}\right)}{L}, \frac{\left(p_{3}-1\right)}{L}\right\},
$$

and $L=\max \left\{L_{1}, G\left(\alpha_{4}\right)\right\}, L_{1}$ is the Lipschitz constant of $G$ on $\left[\alpha_{3}, \alpha_{4}\right]$. Define a map $\mathcal{T}: \mu \rightarrow \mu$ by

$$
\mathcal{T} y(n)=\left\{\begin{array}{l}
\mathcal{T} y\left(n_{1}+\rho\right), n_{1} \leq n \leq n_{1}+\rho \\
\frac{\beta}{p(n+\tau)}-\frac{y(n+\tau)}{p(n+\tau)}+\frac{1}{p(n+\tau)}\left[\sum_{s=n}^{\infty} q(s) G(y(s-\sigma))\right. \\
\left.+\sum_{n_{1} \leq m_{j}-1 \leq n} r\left(m_{j}-1\right) G\left(y\left(m_{j}-\sigma-1\right)\right)\right], n \geq n_{1}+\rho .
\end{array}\right.
$$

For $y \in \mu$ and using (2.2), we have

$$
\begin{aligned}
\mathcal{T} y(n) & \leq \frac{\beta}{p(n+\tau)}+\frac{G\left(\alpha_{4}\right)}{p(n+\tau)}\left[\sum_{s=n}^{\infty} q(s)+\sum_{n_{1} \leq m_{j}-1 \leq n} r\left(m_{j}-1\right)\right] \\
& \leq \frac{1}{p_{3}}\left[\beta+G\left(\alpha_{4}\right) M\right] \\
& =\alpha_{4}
\end{aligned}
$$

and

$$
\begin{aligned}
\mathcal{T} y(n) & \geq \frac{\beta}{p(n+\tau)}-\frac{y(n+\tau)}{p(n+\tau)} \\
& \geq \frac{1}{p_{4}}\left[p_{4} \alpha_{3}+\alpha_{4}-\alpha_{4}\right] \\
& =\alpha_{3}
\end{aligned}
$$


implies that $\mathcal{T} y \in \mu$ for every $n \geq n_{1}$. For $y_{1}, y_{2} \in \mu$, we have

$$
\begin{aligned}
\mid \mathcal{T} y_{1}(n)- & \mathcal{T} y_{2}(n) \mid \\
& \leq \frac{1}{|P(n+\tau)|}\left|y_{1}(n+\tau)-y_{2}(n+\tau)\right| \\
& +\frac{L_{1}}{|P(n+\tau)|} \sum_{s=n}^{\infty} q(s)\left|y_{1}(s-\sigma)-y_{2}(s-\sigma)\right| \\
& +\frac{L_{1}}{|P(n+\tau)|} \sum_{n_{1} \leq m_{j}-1 \leq n} r\left(m_{j}-1\right)\left|y_{1}\left(m_{j}-\sigma-1\right)-y_{2}\left(m_{j}-\sigma-1\right)\right|,
\end{aligned}
$$

that is,

$$
\begin{aligned}
\left|\mathcal{T} y_{1}(n)-\mathcal{T} y_{2}(n)\right| & \leq \frac{1}{p_{3}}\left\|y_{1}-y_{2}\right\|+\frac{L_{1}}{p_{3}}\left\|y_{1}-y_{2}\right\|\left[\sum_{s=n}^{\infty} q(s)+\sum_{n_{1} \leq m_{j}-1 \leq n} r\left(m_{j}-1\right)\right] \\
& \leq \frac{1}{p_{3}}\left\|y_{1}-y_{2}\right\|+\frac{L_{1}}{p_{3}} M\left\|y_{1}-y_{2}\right\| \\
& \leq \frac{1}{p_{3}}\left[1+\frac{p_{3}-1}{2}\right]\left\|y_{1}-y_{2}\right\|
\end{aligned}
$$

implies that

$$
\left\|\mathcal{T} y_{-} \mathcal{T} y_{2}\right\| \leq \lambda\left\|y_{1}-y_{2}\right\|
$$

Therefore, $\mathcal{T}$ is a contraction with $\lambda=\frac{1+p_{3}}{2 p_{3}}<1$. Hence, by Banach's fixed point theorem, $\mathcal{T}$ has a unique point $y \in \mu$ such that $\mathcal{T} y=y$. Therefore,

$$
y(n)=\left\{\begin{array}{l}
y\left(n_{1}+\rho\right), n_{1} \leq n \leq n_{1}+\rho \\
\frac{\beta}{p(n+\tau)}-\frac{y(n+\tau)}{p(n+\tau)}+\frac{1}{p(n+\tau)}\left[\sum_{s=n}^{\infty} q(s) G(y(s-\sigma))\right. \\
\left.+\sum_{n_{1} \leq m_{j}-1 \leq n} r\left(m_{j}-1\right) G\left(y\left(m_{j}-\sigma-1\right)\right)\right], n \geq n_{1}+\rho
\end{array}\right.
$$

and it is easy to see that $y(n)$ is a nonoscillatory solution of $(E)$.

Case-C. $-1<p_{5} \leq p(n) \leq p_{6} \leq 0$

Let $X=l_{\infty}^{n_{0}}$ be the Banach space of real valued bounded functions $y(n)$ for $n \geq n_{0}$ with sup norm defined by $\|y\|=\sup \left\{|y(n)|: n \geq n_{0}\right\}$.

Let $K=\left\{y(n) \in X: y(n) \geq 0\right.$ for $\left.n \geq n_{0}\right\}$. For $y_{1}, y_{2} \in X$ we define $y_{1} \leq y_{2}$ if and only if $y_{2}-y_{1} \in K$. Thus, $X$ is a partially ordered Banach space. Set

$$
\mu=\left\{y \in X: \alpha_{5} \leq y(n) \leq \alpha_{6}, n \geq n_{0}\right\}
$$

where $\alpha_{5}$ and $\alpha_{6}$ are two positive constants such that

$$
\alpha_{5}<\beta<\left(1+p_{5}\right) \alpha_{6} .
$$


From $\left(H_{1}\right)$, it is possible to choose $n_{1}>n_{0}$ such that

$$
\sum_{n=n_{1}}^{\infty} q(n)+\sum_{n_{1} \leq m_{j}-1 \leq n} r\left(m_{j}-1\right)<\frac{\left(1+p_{5}\right) \alpha_{6}-\beta}{G\left(\alpha_{6}\right)}, n \geq n_{1} .
$$

Let $x_{0}(n)=\alpha_{5}$ for $n \geq n_{0}$. Then $x_{0}(n) \in \mu$ and $x_{0}(n)=\inf \mu$. In addition, if $\phi \subset \mu^{*} \subset \mu$, then

$$
\mu^{*}=\left\{y \in X: l_{1} \leq y(n) \leq l_{2}, \alpha_{5} \leq l_{1}, l_{2} \leq \alpha_{6}, n \geq n_{0}\right\}
$$

Let $x_{1}(n)=l_{2}^{\prime}=\sup \left\{l_{2}: \alpha_{5} \leq l_{2} \leq \alpha_{6}\right\}$. Then $x_{1}(n) \in \mu$ and $x_{1}(n)=\sup \mu^{*}$. Since $\mu$ is a closed subset of $X, \mu$ is a complete metric space. Define a map $\mathcal{T}: \mu \rightarrow \mu$ by

$$
\mathcal{T} y(n)=\left\{\begin{array}{l}
\mathcal{T} y\left(n_{1}+\rho\right), n_{1} \leq n \leq n_{1}+\rho, \\
\beta-p(n) y(n-\tau)+\sum_{s=n}^{\infty} q(s) G(y(s-\sigma)) \\
+\sum_{n_{1} \leq m_{j}-1 \leq n} r\left(m_{j}-1\right) G\left(y\left(m_{j}-\sigma-1\right)\right), n \geq n_{1}+\rho .
\end{array}\right.
$$

For $y \in \mu$ and using (2.3), we have

$$
\begin{aligned}
\mathcal{T} y(n) & \leq \beta-p(n) y(n-\tau)+G\left(\alpha_{6}\right)\left[\sum_{s=n}^{\infty} q(s)+\sum_{n_{1} \leq m_{j}-1 \leq n} r\left(m_{j}-1\right)\right] \\
& \leq \beta-p_{5} \alpha_{6}+G\left(\alpha_{6}\right)\left[\frac{\left(1+p_{5}\right) \alpha_{6}-\beta}{G\left(\alpha_{6}\right)}\right] \\
& =\alpha_{6}
\end{aligned}
$$

and

$$
\mathcal{T} y(n) \geq \beta \geq \alpha_{5}
$$

implies that $\mathcal{T} y \in \mu$ for every $n \geq n_{1}$. Let $y_{1}, y_{2} \in \mu$ such that $y_{1} \leq y_{2}$. It is easy to verify that $\mathcal{T} y_{1} \leq \mathcal{T} y_{2}$. Hence, by Knaster-Tarski fixed point theorem, $\mathcal{T}$ has a unique $y \in \mu$ such that $\mathcal{T} y=y$. Therefore,

$$
y(n)=\left\{\begin{array}{l}
y\left(n_{1}+\rho\right), n_{1} \leq n \leq n_{1}+\rho, \\
\beta-p(n) y(n-\tau)+\sum_{s=n}^{\infty} q(s) G(y(s-\sigma)) \\
+\sum_{j=1}^{\infty} r\left(m_{j}-1\right) G\left(y\left(m_{j}-\sigma-1\right)\right), n \geq n_{1}+\rho .
\end{array}\right.
$$

Clearly, $y(n)$ is a nonoscillatory solution of $(E)$.

Case-D. $-\infty<p_{7} \leq p(n) \leq p_{8}<-1$ 
Let $X=l_{\infty}^{n_{0}}$ be the Banach space of real valued bounded functions $y(n)$ for $n \geq n_{0}$ with sup norm defined by $\|y\|=\sup \left\{|y(n)|: n \geq n_{0}\right\}$. Set

$$
\mu=\left\{y \in X: \alpha_{7} \leq y(n) \leq \alpha_{8}, n \geq n_{0}\right\},
$$

where $\alpha_{7}$ and $\alpha_{8}$ are two positive constants such that

$$
-p_{7} \alpha_{7}<\beta<\left(-1-p_{8}\right) \alpha_{8}
$$

Since $\mu$ is a closed subset of $X, \mu$ is a complete metric space. From $\left(H_{1}\right)$, it is possible to choose $n_{1}>n_{0}$ such that

$$
\sum_{s=n}^{\infty} q(s)+\sum_{n_{1} \leq m_{j}-1 \leq n} r\left(m_{j}-1\right)<M, n \geq n_{1} .
$$

where

$$
M=\min \left\{\frac{p_{7} \alpha_{7}+\beta}{L}, \frac{-\left(\beta+\left(1+p_{8}\right) \alpha_{8}\right)}{L}, \frac{\left(-1-p_{8}\right)}{L}\right\}
$$

and $L=\max \left\{L_{1}, G\left(\alpha_{8}\right)\right\}, L_{1}$ is the Lipschitz constant of $G$ on $\left[\alpha_{7}, \alpha_{8}\right]$. For $y \in \mu$ define a map

$$
\mathcal{T} y(n)=\left\{\begin{array}{l}
\mathcal{T} y\left(n_{1}+\rho\right), n_{1} \leq n \leq n_{1}+\rho, \\
-\frac{\beta}{p(n+\tau)}-\frac{y(n+\tau)}{p(n+\tau)}+\frac{1}{p(n+\tau)}\left[\sum_{s=n}^{\infty} q(s) G(y(s-\sigma))\right. \\
\left.+\sum_{n_{1} \leq m_{j}-1 \leq n} r\left(m_{j}-1\right) G\left(y\left(m_{j}-\sigma-1\right)\right)\right], n \geq n_{1}+\rho .
\end{array}\right.
$$

For $y \in \mu$ and using (2.4), we have

$$
\begin{aligned}
\mathcal{T} y(n) & \leq-\frac{\beta}{p(n+\tau)}-\frac{y(n+\tau)}{p(n+\tau)} \\
& \leq-\frac{1}{p_{8}}\left[\left(-1-p_{8}\right) \alpha_{8}+\alpha_{8}\right] \\
& =\alpha_{8}
\end{aligned}
$$

and

$$
\begin{aligned}
\mathcal{T} y(n) & \geq-\frac{\beta}{p(n+\tau)}+\frac{G\left(\alpha_{8}\right)}{p(n+\tau)} \sum_{s=n}^{\infty} q(s)+\sum_{n_{1} \leq m_{j}-1 \leq n} r\left(m_{j}-1\right) \\
& \geq-\frac{1}{p(n+\tau)}\left[\beta-G\left(\alpha_{8}\right)\left[\sum_{s=n}^{\infty} q(s)+\sum_{n_{1} \leq m_{j}-1 \leq n} r\left(m_{j}-1\right)\right]\right] \\
& \geq-\frac{1}{p_{7}}\left[\beta+G\left(\alpha_{8}\right) M\right]
\end{aligned}
$$




$$
\begin{aligned}
& \geq-\frac{1}{p_{7}}\left[\beta-\beta-p_{7} \alpha_{7}\right] \\
& =\alpha_{7}
\end{aligned}
$$

implies that $\mathcal{T} y \in \mu$ for every $n \geq n_{1}$. The remaining part of the proof is similar to that of Case- $\mathrm{B}$, hence it is omitted. This completes the proof of the theorem.

\section{Discussion and an Example}

In [14], Tripathy and Chhatria have studied the oscillatory behaviour of solutions of the system $(E)$ under the sufficient condition

$$
\left(H_{2}\right) \sum_{n=N}^{\infty} q(n)+\sum_{j=1}^{\infty} r\left(m_{j}-1\right)=\infty, N>0
$$

Because of [14, Theorem 2.1], $\left(H_{2}\right)$ may be a necessary and sufficient condition for oscillation of the solutions of the system $(E)$ for all ranges of the neutral coefficient $p(n)$.

We conclude this section with the following example to illustrates our main results.

Example 3.1. Consider the impulsive difference equation of the form

$$
\left(E_{3}\right)\left\{\begin{array}{l}
\Delta\left[y(n)+\frac{1}{e^{n}} y(n-3)\right]+\left(\frac{e-1}{e}\right) e^{-n} y(n-2)=0, n \neq m_{j}, n>3, \\
\triangleq\left[y\left(m_{j}-1\right)+\frac{1}{e^{m_{j}-1}} y\left(m_{j}-4\right)\right]+\left(\frac{e-1}{e}\right) e^{-m_{j}} y\left(m_{j}-3\right)=0, j \in \mathbb{N},
\end{array}\right.
$$

where $\tau=3, \sigma=2, p(n)=\frac{1}{e^{n}}, q(n)=\left(\frac{e-1}{e}\right) e^{-n}, r\left(m_{j}-1\right)=\left(\frac{e-1}{e}\right) e^{-m_{j}}$, $G(u)=u, m_{j}=3 j$ for $j \in \mathbb{N}$. Here

$$
\begin{aligned}
& \sum_{s=3}^{n} q(s)+\sum_{3 \leq m_{j}-1 \leq n} r\left(m_{j}-1\right), n \geq 3 \\
& =\sum_{s=2}^{n}\left(\frac{e-1}{e}\right) e^{-n}+\sum_{2 \leq m_{j}-1 \leq n}\left(\frac{e-1}{e}\right) e^{-m_{j}} \\
& <\infty, \text { as } n \rightarrow \infty .
\end{aligned}
$$

Indeed, all conditions of Theorem 2.1 holds true. Clearly, $y(n)=e$ is a nonoscillatory solution of the first equation of $\left(E_{3}\right)$. It is easy to see that $e$ is a nonoscillatory solution of the second equation of $\left(E_{3}\right)$. 


\section{Acknowledgements}

This work is supported by Rajiv Gandhi National fellowship(UGC), New Delhi, India, through the Letter No. F1-17.1/2017-18/RGNF-2017-18-SC-ORI-35849, dated 11th July, 2017. The author would like to thank the editors and anonymous referees for the careful reading of the manuscript and useful comments which improved the presentation of the paper.

\section{References}

[1] R. P. Agarwal, Difference Equations and Inequalities, Marcel Dekker, New York, 2000.

[2] R. P. Agarwal, M. Bohner S. R. Grace and D. O'Regan, Discrete Oscillation Theory, Hindawi Publishing Corporation, New York, 2005.

[3] G. E. Chatzarakis, P. Gokulraj, T. Kalaimani and V. Sadhasivam, Oscillatory solutions of nonlinear fractional difference equations, Int. J. Difference Equ., 13: 19-31, 2018.

[4] G. E. Chatzarakis, P. Gokulraj and T. Kalaimani, Oscillation tests for fractional difference equations, Tatra Mt. Math. Publ., 71: 53-64, 2018.

[5] V. Lakshmikantham, D. D. Bainov and P. S. Simeonov, Oscillation Theory of Impulsive Differential Equations, World Scientific, Singapore, 1989.

[6] J. Li and J. Shen, Positive solutions for first order difference equations with impulses, Int. J. Difference Equ., 1: 225-239, 2006.

[7] X. Li and Q. Xi, Oscillatory and asymptotic properties of impulsive difference equations with time- varying delays, Int. J. Difference Equ., 4: 201-209, 2009.

[8] Q. L. Li, Z. G. Zhang, F. Gou and H. Y. Liang, Oscillation criteria for third-order difference equations with impulses, J. Comput. Appl. Math., 225: 80-86, 2009.

[9] W. Lu, W. G. Ge and Z. H. Zhao, Oscillatory criteria for third-order nonlinear difference equations with impulses, J. Comput. Appl. Math., 234: 3366-3372, 2010 .

[10] M. Peng, Oscllation theorems for second- order nolnlinear neutral dealy difference equations with impulses, Comput. Math. Appl., 44: 741-749, 2002.

[11] M. Peng, Oscillation criteria for second-order impulsive delay difference equations, Appl. Math. Comput., 146: 227-235, 2003. 
[12] A. M. Samoilenko and N. A. Perestynk, Differential Equations with Impulse Effect, Visca Skola, Kiev, 1987.

[13] A. K. Tripathy and G. N. Chhatria, Oscillation criteria for forced first order nonlinear neutral impulsive difference system, Tatra Mt. Math. Publ., 71: 175-193, 2018.

[14] A. K. Tripathy and G. N. Chhatria, On oscillatory first order neutral impulsive difference equations, Math. Bohem., DOI: 10.21136/MB.2019.0002-18 (online first).

[15] G. P. Wei, The persistance of nonoscillatory solutions of difference equation under impulsive perturbations, Comput. Math. Appl., 50: 1579-1586, 2005.

[16] H. Zhang and L. Chen, Oscillations criteria for a class of second-order impulsive delay difference equations, Adv. Complex Syst., 9: 69-76, 2006. 
